\title{
ANTIMICROBIAL ACTIVITY OF ANISOCHILUS CARNOSUS (L.F.) WALL AGAINST THE HUMAN GASTRIC PATHOGEN HELICOBACTER PYLORI
}

\author{
VIGNESH SHETTY ${ }^{1}$, RICHARD LOBO ${ }^{2}$, NIMMY KUMAR ${ }^{2}$, RAMACHANDRA LINGADAKAI ${ }^{3}$, GANESH C PAI ${ }^{4}$, \\ GIRISH BALARAJU ${ }^{4}$, MAMATHA BALLAL ${ }^{1 *}$
}

${ }^{1}$ Department of Microbiology, Enteric Diseases Division, Central Research Lab, Kasturba Medical College, Manipal University, Manipal - 576 104, Karnataka, India. ${ }^{2}$ Department of Pharmacognosy, Manipal College of Pharmaceutical Sciences, Manipal University, Manipal, Karnataka, India. ${ }^{3}$ Department of Surgery, Kasturba Medical College, Manipal University, Manipal - 576 104, Karnataka, India. ${ }^{4}$ Department of Gastroenterology \& Hepatology, Kasturba Medical College, Manipal University, Manipal - 576 104, Karnataka, India. Email: mamatha.ballal08@gmail.com

Received: 29 May 2017, Revised and Accepted: 01 July 2017

ABSTRACT

Objective: The prevalence of Helicobacter pylori in India is high, and majority leads to severe gastrointestinal infections. Existing treatment regimens for H. pylori infections have increased failure rates and adverse side effects that desire the search for an effective substitute therapy. Anisochilus carnosus (L.f.) wall (Lamiaceae), a herb which grows once in a year at high elevation is used widely in traditional treatment for the complaints of gastric ulcer and skin diseases. The present study was performed to assess the antibacterial activity of $A$. carnosus (L.f.) wall, against clinical isolates of H. pylori in vitro.

Methods: A. carnosus leaves were collected-dried and extracted with water and ethanol by cold maceration with ethanol by soxhlet method. The minimum inhibitory concentration (MIC) of extracts was made and tested against 32 clinical and 1 reference strains of $H$. pylori.

Results: A. carnosus (L.f.) wall inhibited the growth of most of the clinical H. pylori strains. The MIC of A. carnosus (L.f.) wall extracted by cold maceration (aqueous and ethanol) and Soxhlet apparatus (ethanol) ranged from 500 to $62.5 \mu \mathrm{g} / \mathrm{ml}$, and the majority of the clinical H. pylori strains were inhibited at the MIC of $500 \mu \mathrm{g} / \mathrm{ml}$ of aqueous, ethanol, and Soxhlet ethanol extraction were $63.63 \%, 43.75 \%$, and $71.87 \%$, respectively.

Conclusion: A. carnosus (L.f.) wall is an efficient inhibitor of $H$. pylori growth in vitro. A. carnosus (L.f.) wall revealed enormous therapeutic potential to $H$. pylori infection as it was extremely active in the suppression of $H$. pylori. Hence, it can be taken as a potential agent against several $H$. pylori linked gastric pathogenic progressions.

Keywords: Anisochilus carnosus, Helicobacter pylori, Minimum inhibitory concentration, Antimicrobial activity.

(c) 2017 The Authors. Published by Innovare Academic Sciences Pvt Ltd. This is an open access article under the CC BY license (http://creativecommons. org/licenses/by/4. 0/) DOI: http://dx.doi.org/10.22159/ajpcr.2017.v10i10.20304

\section{INTRODUCTION}

Helicobacter pylori is a unipolar flagellated, microaerophilic bacterium which has the capability to survive in human stomach and setup infections that may be able to last for years or decades. One-half or more people worldwide carry these bacteria, as well as its prevalence in developing countries surpasses $90 \%$. The role of H. pylori in causing peptic ulcer disease had attracted greater attention. Indeed, this bacterium is categorized as a carcinogen Group I by the International Agency for Research on Cancer [1-3]. Significant differences among H. pylori strains geographically, by molecular characterization and disease outcome has shown strains from India are genetically different from those from East Asia to Western strains [4,5]. There are numerous recognized virulence-related factors including cag pathogenicity Island (cag PAI), that enhance the pathogenesis and risk of more severe infections like gastric adenocarcinoma [4,6]. However, these H. pylori virulence markers are not always linked with disease, eradication from diseased persons will be the promising/excellent option for an active treatment of $H$. pylori-related infections. A number of combination therapies have been developed for $H$. pylori eradication to treat or prevent infections. However, treatment with the triple therapy is not successful always, due to the acquisition of drug resistance including metronidazole, and clarithromycin which leads to a serious problem for treatment failure [7]. Several studies have demonstrated that the rate of resistance to antibiotics differs geographically, reaching $30-80 \%$ for metronidazole and from $5 \%$ to $30 \%$ in clarithromycin. Understanding the reduced cure rate by conventional therapy due to increasingly resistant strains, adverse side effects, refusal among the patients, the antibiotic regimens cost, and some added factors leading to less effective therapy. Therefore, there is an essential need to progress/ develop novel treatment approaches for $H$. pylori infection [8-10].

A. carnosus (L.f.) wall, an annual herbaceous plant which is a member of the family Lamiaceae, breeds on high elevation amid small rocks. Distribution in India is mainly seen in Karnataka, Maharashtra, Rajasthan, and Tamil Nadu and practiced traditionally in tribal communities for the treatment of ulcer, stomach ache, cough, and eczema. This plant's phytochemical study has revealed it to be rich in active compounds such as saponins, tannins, flavonoids (apigenin and luteolin), phytosterols, triterpenoids, and essential oil components (carvacrol, $\beta$-selinene, camphor, $\alpha$-cis-bergamotene, and caryophyllene) [11-13].

Considering the advantageous background of A. carnosus, the current work has been directed to assess the effectiveness of the A. carnosus (L.f.) wall as an antimicrobial means against $H$. pylori clinical strains obtained from patients in South India.

\section{METHODS}

Collection of plant materials

A. carnosus leaves were collected during September 2014 from rock crevices at Hayagreeva Nagar, Udupi, and it was authenticated by 
Dr. Richard Lobo, Pharmacognosist, Manipal College of Pharmaceutical Sciences (MCOPS), Manipal, Karnataka. A receipt sample with accession number (PP 573) has been placed in the Department of Pharmacognosy, MCOPS, Manipal, India.

\section{Extraction of $A$. carnosus leaves}

The A. carnosus leaves (500 g) were dried, coarsely powdered, and extracted with Soxhlet apparatus using ethanol $(3 \mathrm{~L} \times 1 \mathrm{~L})$. The extracts containing solvent was dried by evaporation on a rotary evaporator kept under low pressure. Dried leaves were crushed (500 g) and kept for cold maceration for 4 days in chloroform:water (1:99) and ethanol at room temperature. The solvent-containing extracts were then filtered and filtrates gained were concentrated to obtain crude aqueous extract and ethanol extract, respectively, on a water bath (Table 1).

\section{Culture and strains of $\boldsymbol{H}$. pylori}

Archived $32 \mathrm{H}$. pylori strains which were isolated from the body and antral mucosal biopsy specimens obtained from patients at the Kasturba Medical College and Hospital, India, who had symptoms of the upper gastrointestinal disorders. Along with the clinical strains, one reference H. pylori strain American Type Culture Collection (ATCC 26695) was used for this study. The identification of the strains was based on colony morphology, gram staining, and biochemical reactions positive for catalase, urease, and oxidase. Revived H. pylori strains were obtained by culturing on brain heart infusion (BHI) agar (Difco Laboratories) supplemented with 7\% horse serum (GIBCO), $0.4 \%$ IsoVitaleX (Becton Dickinson), trimethoprim $(5 \mathrm{~g} / \mathrm{ml})$, vancomycin $(8 \mathrm{~g} / \mathrm{ml})$, and polymyxin $\mathrm{B}(10 \mathrm{~g} / \mathrm{ml})$. The culture plates were incubated in the microaerophilic atmosphere $\left(5 \% \mathrm{O}_{2}, 10 \% \mathrm{CO}_{2}\right.$, and $85 \% \mathrm{~N}_{2}$ ) (double gas incubator; Thermo Fisher Scientific) at $37^{\circ} \mathrm{C}$. The stock cultures were preserved until use at $-80^{\circ} \mathrm{C}$.

\section{Minimum inhibitory concentrations (MIC)}

Stock cultures which were frozen were then cultured on BHI agar supplemented with $7 \%$ horse serum and incubated at microaerophilic conditions for 3 days as noted earlier. Isolates were cultured again on fresh BHI agar plate and incubated for $24 \mathrm{hr}$ at $37^{\circ} \mathrm{C}$. Growing cells of H. pylori were put in sterile phosphate-buffered saline and turbidity adjusted to McFarland Standard 2. Three microliters of the adjusted inoculum were spot inoculated to BHI agar comprising several concentrations of A. carnosus. Dimethyl sulfoxide (Merck Chemical) was used as a solvent for $A$. carnosus extract to dissolve and made a stock concentration of $50 \mathrm{mg} / \mathrm{ml}$. Working concentrations were prepared and are $500 \mu \mathrm{g} / \mathrm{ml}, 250 \mu \mathrm{g} / \mathrm{ml}, 125 \mu \mathrm{g} / \mathrm{ml}$, and $62.5 \mu \mathrm{g} / \mathrm{ml}$ for aqueous, ethanol maceration, and Soxhlet ethanol extraction. For each experiment, growth control plates were included containing only $\mathrm{BHI}$ agar. Incorporating the solvent dimethyl sulfoxide in BHI agar was also considered as growth control to confirm that the viability of the microorganism was not interfered by the solvent used for dissolving extract. 1 reference strain (ATCC 26695) and 32 clinical strains were used for antimicrobial susceptibility testing. Later incubated in the microaerophilic atmosphere at $37^{\circ} \mathrm{C}$ for 5 days. The MIC was welldefined as the lowest concentration of the test compound at which there was no visible growth. Antibiotics clarithromycin (Sigma Chemical Co.) and amoxicillin (Sigma Chemical Co.) were tested for each batch which was considered for quality control and comparative analyses.

\section{RESULTS AND DISCUSSION}

In vitro differential inhibition of $H$. pylori growth by A. carnosus Among $32 \mathrm{H}$. pylori strains which were isolated from patients with gastritis, peptic ulcer, respectively, was tested against $A$. carnosus extract. Out of these 36 strains, 24 strains, including ATCC 26695, showed/tested positive for cagA amplicon and the other 8 showed positive for empty site amplicon, indicative of a lack of the entire cag PAI. Agar dilution method was used to illustrate MICs.

Cold macerations (crude aqueous extract and ethanol extract) of $A$. carnosus against $H$. pylori showed activity with increasing concentration of MIC. The MIC of A. carnosus leaf extract ranged from
$62.5 \mu \mathrm{g} / \mathrm{ml}$ to $500 \mu \mathrm{g} / \mathrm{ml}$, and the majority of the strains inhibited $(63.63 \%$ and $43.75 \%)$ in both aqueous and ethanol extract showed a MIC of $500 \mu \mathrm{g} / \mathrm{ml}$ (Figs. 1 and 2). Soxhlet Ethanol extract of A. carnosus at the MIC $500 \mu \mathrm{g} / \mathrm{ml}$ inhibited the growth of $23(71.87 \%)$ clinical isolates (Fig. 3) (Table 2). The reference strain ATCC 26695 showed activity at MIC $500 \mu \mathrm{g} / \mathrm{ml}$ by all the three extracts.

These findings noticeably confirm that $A$. carnosus can be an effective inhibitor of Indian H. pylori strains growth regardless of the disease

Table 1: Final product obtained after extraction of $500 \mathrm{~g}$ Anisochilus carnosus leaves

\begin{tabular}{ll}
\hline Method & Yield (\%w/w) \\
\hline Aqueous maceration & 0.54 \\
Alcohol (ethanol) maceration & 0.27 \\
Ethanol extract-Soxhlet & 0.34 \\
\hline
\end{tabular}

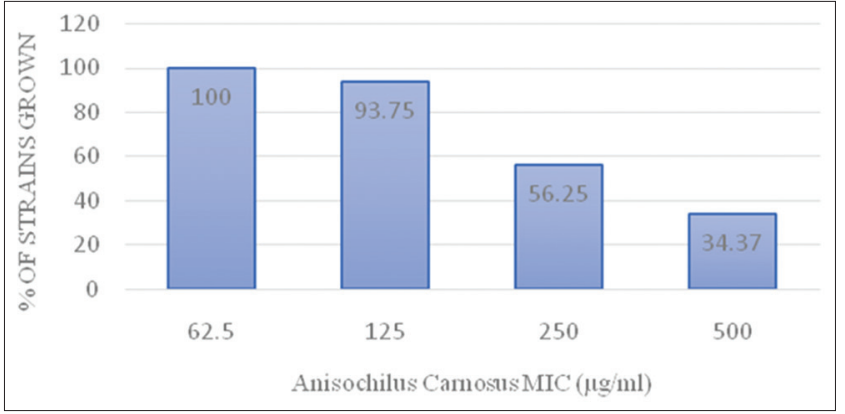

Fig. 1: Helicobacter pylori strains grown in minimum inhibitory concentration of aqueous maceration of Anisochilus carnosus leaves extract

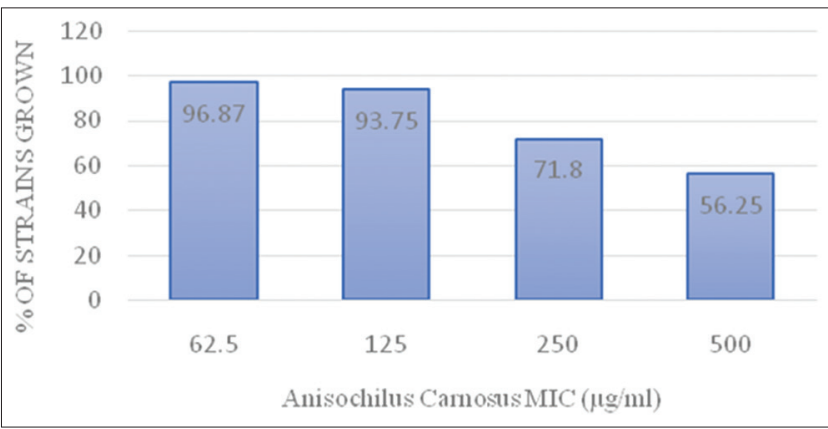

Fig. 2: Helicobacter pylori strains grown in minimum inhibitory concentration of ethanol maceration of Anisochilus carnosus leaves extract

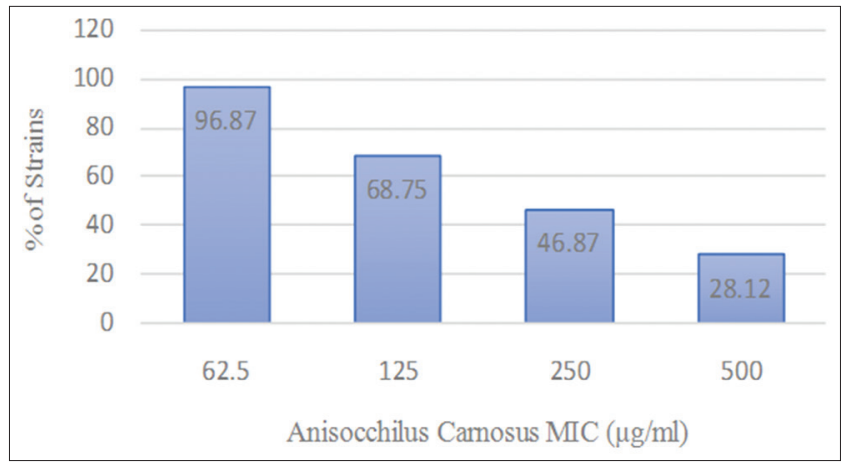

Fig. 3: Helicobacter pylori strains grown in minimum inhibitory concentration of Soxhlet ethanol extract of Anisochilus carnosus leaves 
Table 2: Rate of $\boldsymbol{H}$. pylori clinical strains inhibited against the MIC of $A$. carnosus extract

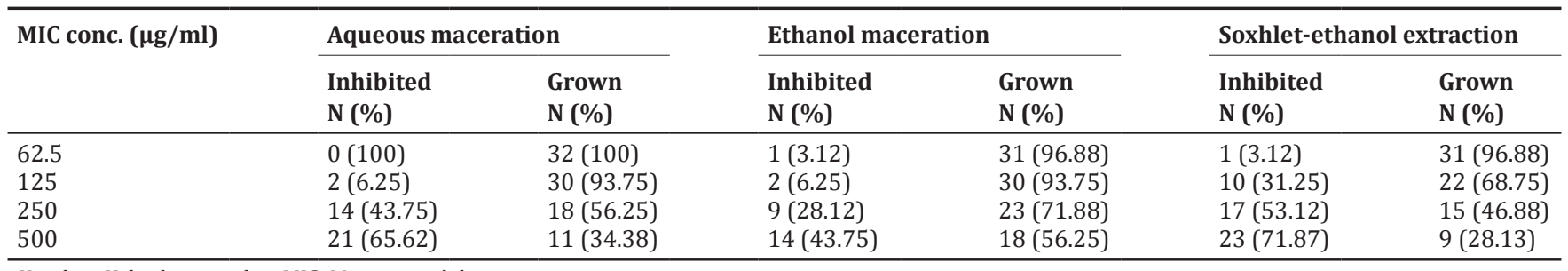

H. pylori: Helicobacter pylori, MIC: Minimum inhibitory concentration

status. Among the three methods followed for extraction of A. carnosus, more promising results were obtained from Soxhlet ethanol extraction. However, further study is needed to observe the strain-specific MIC differences and polymorphism in gene targets.

Several strategies for eradication of H. pylori are followed; among them currently, proton pump inhibitor-based triple therapy, sequential triple therapy is followed for treating H. pylori infection. Despite it having a good rate of success, difficulties such as contraindications and failure in treatment are common in few patients [14-16]. Moreover, quickly developing resistance to the drugs commonly used for treating infections caused by $H$. pylori is yet another hurdle toward achieving an effective eradication therapy [9]. Because of the increasing development of drug resistance by $H$. pylori strains, there is an increasing need to develop an effective, safe and cost-effective therapy which inhibits H. pylori. Traditional system in Indian medicine makes use of treatment based on medicinal plants and their phytochemicals which are effective and are also said to have lesser side effects $[17,18]$. Previous studies on $A$. carnosus have shown that it has potent-anticancer activity and is also traditionally used as a treatment for gastric ulcer and skin diseases. This encouraged us to evaluate its potential antimicrobial activity against $H$. pylori strains isolated from India which has diverse population, i.e., hpAsia2 and hpEurope strains [19]. Moreover, there is a higher prevalence of $H$. pylori in Indian Population with a good number of patients suffering from gastrointestinal disorders [20].

A. carnosus, an annual herb plant which grows more commonly in higher elevation such as rock crevices have shown immense benefits in traditional medicine. Some of the previous studies have specified nanoparticles synthesized from an extract of $A$. carnosus to show effective antimicrobial action against microorganisms such as Escherichia coli, Klebsiella pneumoniae, Bacillus subtilis, and Pseudomonas aeruginosa [21]. Another study, showed A. carnosus to exhibit antiulcer property which has potent gastric healing properties [22]. Few other reports showed an effective hepatoprotective activity by ethanol extract of $A$. carnosus [23]. In addition, leaves of $A$. carnosus presented good antipyretic and analgesic activity [24]. A recent study in India studied the cytotoxic potential of A. carnosus [12]. This suggests that $A$. carnosus contain strong pharmacological properties, which could probably be attributed to the phytochemicals present in it, making it an important medicinal plant.

\section{CONCLUSION}

In this study, we have examined the A. carnosus activity against 32 clinical strains which were isolated from patients suffering from gastrointestinal infections. A. carnosus extract inhibited H. pylori strains at MIC $500 \mu \mathrm{g} / \mathrm{ml}$. The anti- $H$. pylori activity is irrespective of the virulence genotypes harbored by strains observed by checking cagPAIpositive and cagPAI-negative strains. It is noteworthy to be considered as a potential antimicrobial agent for the eradication of $H$. pylori especially due to the development of increasing drug resistance by $H$. pylori strains. Our findings prompt for further studies to elucidate the mechanism by which $A$. carnosus inhibits $H$. pylori growth. All these observations along with the previous study suggest $A$. carnosus possess potent-antimicrobial activity, antiulcer, and anticancer activity. All these important data can be taken into consideration for developing an effective alternative therapy for H. pylori. However, further studies are needed to extrapolate its effect in vivo on animal and humans.

\section{REFERENCES}

1. Covacci A, Telford JL, Del Giudice G, Parsonnet J, Rappuoli R. Helicobacter pylori virulence and genetic geography. Science 1999;284(5418):1328-33.

2. Shetty V, Ballal M, Lingadakai R, Mukhopadhyay AK. Determination of Helicobacter pylori virulence genes in clinical isolates of symptomatic patients from South Coastal region of Karnataka - A preliminary work. Austin J Gastroenterol 2015;2(1):1031.

3. Correa P, Piazuelo MB. Helicobacter pylori infection and gastric adenocarcinoma. US Gastroenterol Hepatol Rev 2011;7(1):59-64.

4. Yamaoka Y. Mechanisms of disease: Helicobacter pylori virulence factors. Nat Rev Gastroenterol Hepatol 2010;7(11):629-41.

5. Sgouros SN, Bergele C. Clinical outcome of patients with Helicobacter pylori infection: The bug, the host, or the environment? Postgrad Med J 2006;82(967):338-42.

6. Roesler BM, Rabelo-Gonçalves EM, Zeitune JM. Virulence factors of Helicobacter pylori: A review. Clin Med Insights Gastroenterol 2014;7:9-17.

7. Toracchio S, Cellini L, Di Campli E, Cappello G, Malatesta MG, Ferri A, et al. Role of antimicrobial susceptibility testing on efficacy of triple therapy in Helicobacter pylori eradication. Aliment Pharmacol Ther 2000;14(12):1639-43.

8. Georgopoulos SD, Papastergiou V, Karatapanis S. Helicobacter pylori eradication therapies in the era of increasing antibiotic resistance: A paradigm shift to improved efficacy. Gastroenterol Res Pract 2012;2012:757926.

9. Ghotaslou R, Leylabadlo HE, Asl YM. Prevalence of antibiotic resistance in Helicobacter pylori: A recent literature review. World J Methodol 2015;5(3):164-74.

10. Ashwini P, Sumana MN, Shilpa U, Mamatha P, Manasa P, Dhananjaya BL, et al. A review on Helicobacter pylori: Its biology, complications and management. Int J Pharm Pharm Sci 2015;7 Suppl 1:14-20.

11. Lobo R, Bhagat J, Ballal M, Gupta N. Histo-anatomical study of Anisochilus carnosus (Lf) wall: An Indian habitant. Res J Med Plant 2012;6(6):456-60.

12. Bhagat J, Lobo R, Kumar N, Mathew JE, Pai A. Cytotoxic potential of Anisochilus carnosus (L.f.) Wall and estimation of luteolin content by HPLC. BMC Complement Altern Med 2014 28;14:421.

13. Senatore F, Lentini F, Venza F, Bruno M, Napolitano F. Composition and antibacterial activity of the essential oil of Anisochilus carnosus (Linn. fil.) Benth., a Tamil plant acclimatized in Sicily. Flavour Fragr J 2003;18(3):202-4.

14. Vaira D, Zullo A, Hassan C, Fiorini G, Vakil N. Sequential therapy for Helicobacter pylori eradication: The time is now! Therap Adv Gastroenterol 2009;2(6):317-22.

15. Scaccianoce G, Hassan C, Panarese A, Piglionica D, Morini S, Zullo A. Helicobacter pylori eradication with either seven-day or 10-day triple therapies, and with a 10-day sequential regimen. Can J Gastroenterol 2006;20(2):113-7.

16. Olokoba AB, Obateru OA, Bojuwoye MO. Helicobacter pylori eradication therapy: A review of current trends. Niger Med J 2013;54(1):1-4.

17. Pandey MM, Rastogi S, Rawat AK. Indian traditional ayurvedic system of medicine and nutritional supplementation. Evid Based Complement Alternat Med 2013;2013:376327.

18. Raj T, Kumar P, Rathee R, Dubey KK. Screening of some medicinal plants for their antimicrobial activities. Int J Pharm Pharm Sci 2016;8(5):202-6. 
19. Devi SM, Ahmed I, Francalacci P, Hussain MA, Akhter Y, Alvi A, et al. Ancestral European roots of Helicobacter pylori in India. BMC Genomics 2007;8(1):184.

20. Thirumurthi S, Graham DY. Helicobacter pylori infection in India from a Western perspective. Indian J Med Res 2012;136(4):549-62.

21. Syed A, Vijaya PP, Saranya RS, Yogananth N, Anurath A, Parveen PK. Antimicrobial activity and characterization of biosynthesized silver nanoparticles from Anisochilus carnosus. Int J Nano Dimens 2013;4:255-62.

22. Mohammed A, Kumar RJ, Santosh HY, Nagashruthi MH. Antiulcer activity of Anisochilus carnosus leaf extracts in pylorus ligation rats. Indian Drugs 2008;45(12):979-81.

23. Yadav R, Yadav AK, Mishra PK, Goswami S, Rao CV. Hepatoprotective and antioxidant activity of ethanolic extract of Anisochilus carnosus whole plant against paracetamol induced liver injury. J Chem Pharm Res 2016;8(8):1176-81.

24. Porika V, Dinakar A, Senthilkumar N. Evaluation of hepatoprotective, analgesic and antipyretic activity of aqueous extracts of Boerhavia diffusa and Anisochlilus carnosus. Int J Pharm Bio Sci 2013;4(1):596-603. 